\title{
AH-7921: the list of new psychoactive opioids is expanded
}

\author{
Maria Katselou • Ioannis Papoutsis • \\ Panagiota Nikolaou • Chara Spiliopoulou • \\ Sotiris Athanaselis
}

Received: 15 December 2014/ Accepted: 8 February 2015/Published online: 21 February 2015

(C) Japanese Association of Forensic Toxicology and Springer Japan 2015

\begin{abstract}
AH-7921 is a structurally unique synthetic opioid analgesic that has recently entered the drug arena in Europe, the USA, and Japan. Although it was synthesized and patented in the mid-1970s, it was first identified in a seized sample purchased via the Internet in July 2012 and formally brought to the attention of the European Union early warning system in August 2012 by the United Kingdom. Several in vitro experiments and animal model studies established the morphine-like analgesic action of AH-7921 as a $\mu$-opioid receptor agonist that has been found to be several times more potent than codeine and at least as potent as morphine. This novel psychoactive substance has already led to eight non-fatal intoxications and 16 deaths in Sweden, the United Kingdom, Norway, and the USA. Thus, AH-7921 is a current public health risk, and better international collaboration, effective legislation and continuous community alertness are needed to tackle this current growing problem. The aim of this review is to summarize the current knowledge about this drug concerning its chemistry, pharmacology, and toxicology, as well as its international legal status. The limited existing analytical methodologies for the determination of AH-7921 in biological samples are also presented. Published or reported AH-7921-related cases, fatalities, or intoxications, and self reports from drug users are reviewed.
\end{abstract}

M. Katselou · I. Papoutsis · P. Nikolaou ( $\square)$.

C. Spiliopoulou $\cdot$ S. Athanaselis

Department of Forensic Medicine and Toxicology, Faculty of

Medicine, National and Kapodistrian University of Athens, 75

Mikras Asias, 11527 Athens, Greece

e-mail: pan_nik@hotmail.com
Keywords AH-7921 - Doxylam - New psychoactive substance $\cdot \mu$-Opioid receptor agonist $\cdot$ Pharmacology and toxicology

\section{Introduction}

New psychoactive substances (NPSs) are continuously emerging on the recreational and illicit drug market. They are often referred to as "legal highs", "designer drugs", or "bath salts" [1-3]. They are mainly synthetic derivatives and analogues of existing controlled drugs, analogues of pharmaceutical products, herbs, fungi, and their extracts that show psychoactive actions. Some NPSs are controlled, while others are legally sourced and can either be sold directly on the drug market or be used as precursors for the synthesis of other designer drugs that mimic the psychoactive effects of controlled substances [4, 5]; thus, due to the chemical alterations of the parent compounds, NPS derivatives can avoid drug legislation. Furthermore, for most NPSs, pharmacological and analytical data are limited or unavailable, which makes detection, monitoring, and control more difficult.

AH-7921 is a new, structurally atypical synthetic opioid analgesic that appears to be sold as a "research chemical" or "legal opioid" on the Internet since 2012. It was synthesized in the 1970s by Allen and Hanburys Ltd. as a potential analgesic medicine; however, its development was abandoned due to its addictive properties. It has never been marketed as a medicine, nor used as pharmaceutical or medicinal product; it has also no industrial use [6]. There are very few references available on this compound [7]. In vivo studies in animals indicated its $\mu$-opioid receptor agonistic activities, although no studies have evaluated its pharmacological and toxicological properties 
in humans. Its activities are similar to those of morphine and include analgesia, hypothermia, respiratory depression, and addictive behavior [7-9]. The abuse of AH-7921 has been reported in eight member states of the European Union as well as in Norway, leading to severe toxicity (non-fatal) cases and 16 reported deaths within a limited period of time (December 2012-September 2013). Thus, AH-7921 is controlled in six European countries (Sweden, Poland, Romania, Finland, Netherlands, and Norway) since 2013 [6].

The aim of this review is to summarize the current knowledge about AH-7921 concerning its chemistry, pharmacology, and toxicology, as well as its legal status. The limited existing analytical methodologies for determination of AH-7921 in biological samples are also presented. Published or reported AH-7921-related cases, fatalities, intoxications, and self reports from drug users are reviewed.

\section{Chemistry}

AH-7921 is an $N$-substituted cyclohexylmethylbenzamide, where the benzamide moiety is dichlorinated at positions 3 and 4 of the benzyl ring, and the aminocyclohexane moiety is $N, N$-dimethylated. "AH" refers to "Allen and Hanburys", the company that patented the drug. Its systematic (International Union of Pure and Applied Chemistry) name is 3,4-dichloro- $N$-\{[1-(dimethylamino)cyclohexyl]methyl $\}$ benzamide, but it can also be found as 1-(3,4-dichlorobenzamidomethyl)cyclohexyldimethylamine $[6,10]$. Common names like doxylam, doxylan, and CN 292429 98 (CAS Number) are also used [6]. The chemical structure of AH-7921 is shown in Fig. 1.

AH-7921 is an off-white solid with the molecular formula $\mathrm{C}_{16} \mathrm{H}_{22} \mathrm{Cl}_{2} \mathrm{~N}_{2} \mathrm{O}$. Its molecular weight is $329.26 \mathrm{~g} / \mathrm{mol}$, and the melting point of its hydrochloride salt is $215-216^{\circ} \mathrm{C}$. It is soluble in ethanol $(11 \mathrm{mg} / \mathrm{ml})$, dimethylsulfoxide $(3 \mathrm{mg} / \mathrm{ml})$ and dimethylformamide $(10 \mathrm{mg} /$ $\mathrm{ml}$ ). The drug absorbs at 205 and $237 \mathrm{~nm}$ spectrophotometrically [10].

\section{Prevalence and use}

AH-7921 appeared on the European drug market in 2012. However, in July 2012 it was formally brought to the attention of the European Union early warning system for the first time by Norway and Sweden, and later, in August 2012, by the United Kingdom. In 2013, it was identified as a co-ingredient in synthetic cannabinoid- and cathinone-derivative products sold in Japan [11]. AH-7921 is sold via the Internet on various websites where it is sometimes referred to as "doxylam", which is its alternative street name, and is also used in Internet drug forums, "legal high" websites, and head shops. This name may be confused with "doxylamine", the International Nonproprietary Name of a widely used (non-prescription) antihistaminic medicine with sedative-hypnotic properties that is chemically completely different from AH-7921 (Fig. 1). Thus, intake of AH-7921 mislabeled as "doxylamine" could lead to unintentional toxicity or overdoses with the drug.

AH-7921 has no established or acknowledged industrial, agrochemical, cosmetic, human, or veterinary medical value or use, and thus there is neither marketing authorization for this NPS in the European Union nor in the 28 member states, Norway, and Iceland. AH-7921 is legitimately used as an analytical reference standard and in scientific research $[6,12,13]$, and can be ordered online from suppliers of chemicals. These licit products are, of course, not intended for human consumption. Relative labeling ("analytical reference standard", "laboratory reagent only", or "not for human consumption") appears on some of the dubious products that are sold through the Internet on different websites to conform to law [6].

According to the EMCDDA, AH-7921 collected from street samples or drug seizures was in powder form; however, it is not known whether the salt or free base forms were present [6]. Internet licit retailers offer AH-7921 for sale in the form of free base or as its hydrochloride salt [12]. AH-7921 was first detected in the United Kingdom in July 2012 in a collected sample of $250 \mathrm{mg}$ of a white powder purchased for $10 \mathrm{GBP}$ via the Internet. Various quantities of powder ranging from 0.02 (Sweden) to $500 \mathrm{~g}$ (France) were seized between July 2012 and October 2013. By March 2014, Europol received reports related to the detection of AH-7921 in seized or collected materials at the scene of death or in biological fluids from Austria, Belgium, Denmark, Finland, France, Germany, Norway, and Sweden $[6,14,15]$.

Information about the dosage of AH-7921, the route of administration, its purity when purchased (mainly online) from drug retailers, and its concomitant use with other substance(s), such as medicine(s) or alcohol, can be extracted from self-reported experiences in drug forums or other related websites. The drug is consumed by nasal insufflation, sublingual application, intravenous injection, a combination of insufflation and oral consumption, or rectal administration (in the form of powder, tablet, or capsule) at doses ranging from 10 to $150 \mathrm{mg}$ [10, 15-18]. An AH7921 user reports that "The threshold dose for me was around $0.05 \mathrm{~g}$. I explored doses up to $0.512 \mathrm{~g}$. I also experimented with immediate tolerance using multiple doses. I found that re-dosing between 1 a.m. and 3 a.m. produced the smoothest increase in effects and duration" [17]. 
Many users take AH-7921 in combination with other psychoactive substances and/or controlled drugs, especially cannabis $[16,17]$. In some fatal or intoxication cases reported by the member states and Norway, medicines, controlled drugs, and/or other NPSs were also detected in biological samples along with AH-7921 [14].

\section{Pharmacology and toxicology}

AH-7921 was designed, synthesized, developed, and patented in the mid 1970 s by researchers from the University of Aston (Birmingham) and the pharmaceutical company Allen and Hanburys Ltd. It was synthesized among other $N$-substituted cyclohexylmethylbenzamide derivatives as a potential analgesic agent due to its structural similarity to fentanyl and phencyclidine (Fig. 1). AH7921 proved to be the most active compound of the designed series in animal models tested possessing significant analgesic properties $\left(\mathrm{ED}_{50}=0.55 \mathrm{mg} / \mathrm{kg}\right)$. Especially in mouse, AH-7921 showed an analgesic action equal to morphine $\left(\mathrm{ED}_{50}=0.45 \mathrm{mg} / \mathrm{kg}\right)$ when inhibiting phenylquinone-induced writhing [8]. After subcutaneous administration in mice, the drug proved to be an antinociceptive agent during hot-plate test [19].

Because early in vivo animal studies of AH-7921 revealed its potent analgesic activity, it was selected among the series of $N$-substituted cyclohexylmethylbenzamides for further investigation and detailed studies in higher species. Particularly, orally administered doses of AH-7921 in conscious dogs led to suppression of pain response (to electrical stimulation of the dental pulp) to an extent similar to that of morphine with a minimal effective antinociceptive oral dose of $1.25 \mathrm{mg} / \mathrm{kg}$. The corresponding analgesic dose for codeine was $3.5 \mathrm{mg} / \mathrm{kg}$. In rhesus monkeys, the minimal oral antinociceptive (for similarly induced pain) doses of AH-7921, codeine, and morphine were $13.8,11.3$, and $\leq 5.0 \mathrm{mg} / \mathrm{kg}$, respectively. These doses caused no overt behavioral effects in the above examined animal models, although higher oral doses of $50 \mathrm{mg} / \mathrm{kg}$ caused slight central nervous system depression. Further studies by the same scientific group of Allen and Hanburys Ltd. revealed the addictive liability of AH-7921; administration of naloxone to rats that were orally treated with $5 \mathrm{mg} \mathrm{AH}-7921 / \mathrm{kg}$ three times daily that increased to $20 \mathrm{mg} \mathrm{AH}-7921 / \mathrm{kg}$ three times daily over 5 days caused an withdrawal syndrome similar to that produced in animals that had received morphine on a similar dosage schedule. The symptoms were completely alleviated after administration of certain doses of $\mathrm{AH}$ 7921. In addition, on terminating AH-7921 treatment, withdrawal signs appeared over a period of 24-48 h, which indicates that the physical dependence produced is similar to that of opioids [20].

Sewell and Spencer [21] in their study showed that the antinociceptive effects of subcutaneously injected morphine or AH-7921 were prolonged in both cases by intracerebroventricularly injecting serotonin in mice, while noradrenaline attenuated the antinociceptive effects of these two analgesics. Thus, it was assumed that AH-7921 interacts in vivo with brain-penetrating serotonergic and adrenergic drugs.
Fig. 1 Chemical structures of AH-7921, fentanyl, doxylamine, and phencyclidine

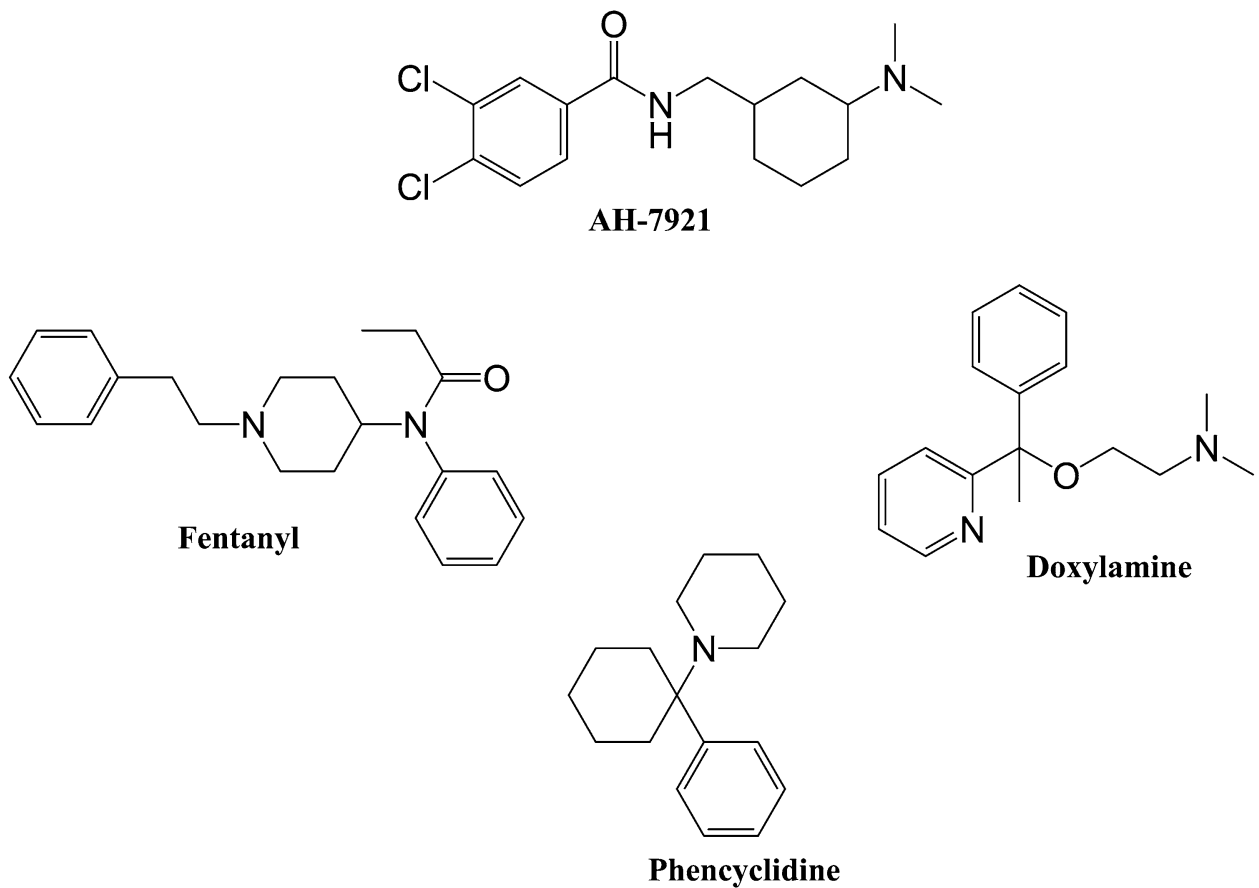


More recent studies in rodents indicated that AH-7921 was a $\mu$-opioid receptor agonist, while $\kappa$-opioid receptors were also involved to its analgesic effect against chemically induced pain [19]. This moderate selectivity towards $\mu$ - over $\kappa$-opioid receptors was also confirmed in vitro using opioid receptor preparations from guinea pig brains [22]. Hayes and Tyers [8] studied the antinociceptive effects, as well as the adverse effects produced by $\mathrm{AH}-$ 7921 and other selected opioid receptor agonists, after subcutaneous administration in mice. These adverse effects included miosis, Straub tail response, hypothermia, sedation, respiratory depression $\left(\mathrm{ED}_{50}=2.5 \mathrm{mg} / \mathrm{kg}\right)$, and inhibition of gastrointestinal propulsion. In general, these effects of AH-7921 were similar to those of morphine, indicating a shared mode of action at the receptor level, while they were significantly reduced by simultaneous subcutaneous administration of naloxone. These results demonstrated that the doses that produced side effects were close to those responsible for its analgesic action. In the same study, morphine was found to have a twofold greater safety margin than AH-7921, which suggests easier appearance of toxic effects after AH-7921 use [8].

There are no published studies concerning the pharmacological or toxicological action of AH-7921 in humans and the clinical features of its acute toxicity [7]. Most of that information comes from drug users. Thus, the only available information related to the use and the effects of this drug is scarce and originates from Internet drug forums and a limited number of recently published poisoning cases. AH-7921 as a $\mu$-opioid receptor agonist produces relaxation, euphoria, respiratory depression, nausea, hypertension, and hypothermia, as users of this drug have mentioned $[6,10]$. However, it is difficult to estimate the role of $\kappa$-opioid receptor agonism in the overall physiological and psychological effects of AH-7921 in humans. Drug users reported that the desired (positive) effects of AH-7921 after oral intake include physical anesthetic effect, euphoria, mental relaxation, and pleasant mood lift, while nausea and vertigo induced by movement appeared as adverse (negative) effects that could be minimized by having a meal $2-4 \mathrm{~h}$ prior to ingestion. According to a user who had been in search of a substance that would provide pain management and tried AH-7921 orally, "The effects of AH-7921 seem to be highly predictable and repeatable. Nearly every experience exhibited the same basic effects on my mental and physical being. I found the intensity of the effects to correspond in a linear relationship to the dosage amount" [17].

An AH-7921 user mentioned that the inhalation of vapors in an amount of $40 \mathrm{mg}$ as free base led to a very relaxing and pleasant experience, euphoria, and pain relief similar to those produced by doses of morphine as low as $10 \mathrm{mg}$. The peak-effects lasted for $1.5 \mathrm{~h}$. No other effects except warmness of body, extreme relaxation, analgesic effects, medium euphoria, and miosis (occurring with the use of other opioids) were noticed [16]. Two AH-7921 users experienced similar effects as well as an addition "opiate glow", alertness, occasional itching, nausea, and tremors after sublingual administration and re-dosing of a solution of powder AH-7921 in lemon juice and warm water [23]. No serious adverse effects were observed by these users.

A user who consumed orally in total $2 \mathrm{~g}$ of AH-7921 in 3 weeks experienced feelings of depression and mild insomnia after withdrawal from the drug, while his second experience resulted in a visit to hospital with "numbness, spasms, and twitches" [18]. Other users have confirmed that AH-7921 can lead to withdrawal symptoms worse than morphine due to its much longer half-life [24].

\section{Intoxication and fatal cases related to AH-7921 use}

The growing recreational use of NPSs has led to an increasing number of confirmed cases of severe intoxications and fatalities associated with their consumption in Europe and worldwide [14]. According to the EMCDDA report of 2014, the Swedish Poison Information Centre reported eight non-fatal intoxications associated with $\mathrm{AH}-$ 7921 use between December 2012 and April 2013. The presence of AH-7921 was analytically confirmed in seven of these cases, but no information on AH-7921 quantification was given. The clinical symptoms described in the above cases included seizures, hypertension, and tachycardia $[6,14,15]$.

The first death associated with AH-7921 was reported by Norway in December 2012. Since then, a total of 16 deaths in which AH-7921 was detected have been reported from Sweden, the United Kingdom, and Norway [6, 14, 15].

Three fatal cases, in which the use of AH-7921 was involved, occurred in Norway between December 2012 and August 2013. In the first case of the three, AH-7921 was not analytically confirmed in biological samples, but its use was suspected due to the detection of AH-7921 in the small amounts of a white powder found in a bag and in a syringe with dried blood at the location close to the deceased during the investigation at the scene of death. Etizolam and phenazepam were also found in the bag and the syringe. In the second case, a 23-year-old male probably bought drugs via the Internet and overdosed. AH-7921 was detected in his peripheral blood at a concentration of $0.428 \mu \mathrm{g} / \mathrm{ml}$. 2-Fluoromethamphetamine, 3-methylmethcathinone, codeine, and paracetamol were also detected. In the third case, the death was associated with AH-7921 in Norway, but the drug was analyzed in Sweden. AH-7921 was quantified in femoral 
blood at a concentration of $0.34 \mu \mathrm{g} / \mathrm{ml}$. Etizolam was also detected $[6,14,15]$.

Three deaths associated with AH-7921 were also reported in the United Kingdom between February 2013 and August 2013. The involvement of this NPS was analytically confirmed [25]. In the first case (February 2013), AH-7921 was detected in postmortem femoral blood at a concentration of $0.58 \mu \mathrm{g} / \mathrm{ml}$. In addition to AH-7921, etizolam, etaqualone, mephedrone, pentedone, diphenylpropinol, and 4-methylmethcathinone were detected in postmortem urine. AH-7921 was also detected in a packet of labeled powder found at the scene of death. In the second case (July 2013), AH-7921 was determined at a concentration of $0.05 \mu \mathrm{g} / \mathrm{ml}$ in the femoral blood of a man found dead with a bag containing chloroform over his head. Chloroform and ethanol were also detected from his blood. In August 2013, the victim of the third case was found unresponsive at home and died later after being transferred to a hospital. In postmortem femoral blood, AH-7921 $(4.46 \mu \mathrm{g} / \mathrm{ml})$, mirtazapine, and doxylamine were detected. Their presence was also confirmed in urine along with clobazam and a codeine metabolite $[6,14,15]$.

Ten lethal cases were reported in Sweden between January and September 2013, and all cases were analytically confirmed. In nine of them, AH-7921 was determined in femoral blood at concentrations ranging from 0.03 to $0.99 \mu \mathrm{g} / \mathrm{ml}$, while in the tenth case it was detected postmortem in hair although not quantified. In all cases, at least one other psychoactive substance was detected during the toxicological analysis. These substances included amphetamine (two cases), 3-methylmethcathinone (two cases), a metabolite of ketamine, alcohol, buprenorphine, and drugs such as benzodiazepines (alprazolam, diazepam, nordazepam, pyrazolam), pregabalin, gabapentin, zopiclone, mirtazapine, paroxetine, bupropion, and aripiprazole. In three cases, the death was attributed to "toxic effect of AH-7921" or "overdose of AH-7921", while in four cases, the causes of death were given as "unintentional overdose", "overdose of benzodiazepines and opiates", "intoxication with opioids among others", and "pneumonia caused by aspiration". In two cases, the cause of death was reported as "unclear", and in the last case, no further information was provided $[6,9,14,15]$.

Vorce et al. [26] reported a death in the USA associated with AH-7921 of a 19-year-old male found dead in bed by his friend. The latter indicated that the deceased had purchased two vials of an unknown drug and used them for two consecutive nights. He died during the second night. Pulmonary congestion, edema of the lungs, and enlargement of the liver and spleen were revealed during autopsy. The analysis of the content of the two vials found at the scene identified AH-7921 in one vial and 4-methyl- $\alpha$-pyrrolidinohexanophenone (MPHP) in another vial. In the postmortem blood, only AH-7921 was detected and quantified, while dextromethorphan and MPHP were found in the postmortem urine along with AH-7921. The death was attributed to AH-7921 intoxication. The drug was obviously taken at the night of his death.

\section{Analysis of AH-7921 in seized materials and biological samples}

Because of the chemically basic nature and structure of AH-7921, its extraction from biological samples and analysis are simple, easy, and thus amenable to a number of techniques.

Soh and Elliot [27] reported the development of highperformance liquid chromatography-diode array (HPLC-DAD) detection, liquid chromatography-tandem mass spectrometry (LC-MS-MS), and ultra high-performance liquid chromatography-quadrupole time-offlight-mass spectrometry methods for determination of AH-7921 and its metabolites simultaneously with other NPSs and their metabolites in blood and plasma samples. AH-7921 was liquid-liquid extracted with 1-chlorobutane after alkalization of the biological samples. The developed methods were applied for forensic purposes and were also used to study the stability of these emerging substances and their metabolites in the biological fluids [27].

Uchiyama et al. [11] detected and identified AH-7921 in an illegal product in Japan based on previously published gas chromatography-mass spectrometry (GC-MS) and ultra performance liquid chromatography-electronspray ionization-mass spectrometry techniques after extraction with methanol from herbal-type products crushed to powder [28].

Vorce et al. [26] developed an analytical method using GC-MS for detection and quantification of AH-7921 during toxicological analysis of biological samples. The isolation of AH-7921 from postmortem blood was succeeded by liquid-liquid extraction with $n$-butyl chloride from alkalinized samples. The analyses were based on the use of GC-MS using selected ion monitoring mode; the electron impact (EI) mass spectrum of the underivatized AH-7921 showed characteristic fragments at $\mathrm{m} / \mathrm{z} 126$ (base peak), 145, and 173. Two other substances were also detected. Their characteristic fragments were $m / z, 112$ (base peak), 145 , and 173, and $m / z 98$ (base peak), 145, 173, respectively. They were suggested to be the AH-7921 metabolites, $N$-desmethyl-AH-7921 and N,N-didesmethyl-AH7921, produced by the demethylation and di-demethylation of the $\mathrm{N}, \mathrm{N}$-dimethylcyclohexanamine group, respectively [26]. However, the absence of metabolic studies and certified reference standards precluded the verification of the 
other detected substances as metabolites. The possibility also exists that these substances could be contaminants or byproducts found in the material.

Nuclear magnetic resonance and Fourier transform infrared spectra of AH-7921 are available at European information system and database on new drugs and could be used for the identification of the drug in seized materials [10, 29-31].

\section{Legal status}

AH-7921 is currently controlled in six European countries (Sweden, Poland, Romania, Finland, Netherlands, and Norway) [6]. Particularly, in Sweden it is controlled under the Narcotic Drugs Control Act (SFS 1992-860) and the Narcotic Drugs Control Ordinance (SFS 1994-1554) complying with the obligations of the 1971 United Nations Convention on Psychotropic Substances. In Poland, AH7921 fell under the definition of a "substitution" drug under the act amending the Act on State Sanitary Inspection (2010), and thus its production and marketing are penalized with administrative sanctions (i.e. fine). In Romania it is controlled under Law 194/2011 as a substance with psychoactive potential until proven harmless. Netherlands and Finland reported that AH-7921 is a controlled substance and must comply with medicine legislation (in Finland since 15 March 2013-Medicines Act 395/87). In Norway it is regulated by the Medicines Act, and is available only by prescription. There is no information regarding the control status of AH-7921 in Spain. The remaining 22 Member States (Austria, Belgium, Bulgaria, Croatia, Cyprus, the Czech Republic, Denmark, Estonia, France, Germany, Greece, Hungary, Ireland, Italy, Latvia, Lithuania, Luxembourg, Malta, Portugal, Slovakia, Slovenia, and the United Kingdom) and Turkey reported that AH-7921 is not controlled at the national level, although Germany intended to introduce national measures for this NPS [6, 14, 15, 29, 32]. In June 2014, the European Commission proposed to member states to take the necessary measures to subject AH-7921, along with other NPSs, to control to prevent its further spread [32]. Following this proposal, a decision on scheduling AH-7921 in the member states was taken in September 2014 [6]. The distribution of AH-7921 has been considered to be illegal in Israel since 12 September 2013 [33]. In Australia, AH7921 was scheduled as a Prohibited Substance (subjected to Schedule 9 of the Standard for the Uniform Scheduling of Medicines and Poisons) in May 2014, although this amendment has been repealed as of 2 June 2014 [34]; but its import is still banned. It is expected that AH-7921 will be a controlled substance in most countries worldwide by the end of 2015.

\section{Conclusions}

AH-7921 is an NPS available online and constitutes a public health danger in many countries worldwide. It is a potential analgesic drug with addictive properties and has been synthesized to mimic the effects of morphine, fentanyl, and phencyclidine due to structural similarity to the latter two, and at the same time, it outsmarts the drug laws. AH-7921 was first synthesized in the mid-1970s, but it only recently (in 2012) appeared on the drug market and gained the attention of authorities due to the intoxications and fatalities it caused. It is considered a synthetic opioid, and its pharmacological properties are similar to morphine. Potential side effects include miosis, hypothermia, sedation, respiratory depression, and inhibition of gastrointestinal propulsion, as well as, in severe cases, death. The drug is available online in the powder form, and is often used concomitantly with other recreational drugs. In 2012 and 2013, a number of reported deaths associated with the intake of AH-7921 led to control of this substance in six European countries, Israel, and Australia. In September 2014, the European Commission published a decision calling upon its member states to take the necessary measures to subject AH-7921, along with other NPSs, to control to prevent its further spread. Similar actions are expected to be taken worldwide.

Conflict of interest There are no financial or other relations that could lead to a conflict of interest.

\section{References}

1. Kikura-Hanajiri R, Uchiyama N, Kawamura M, Goda Y (2013) Changes in the prevalence of synthetic cannabinoids and cathinone derivatives in Japan until early 2012. Forensic Toxicol 31:44-53

2. Nieddu M, Trignano C, Burrai L, Pirisi MA, Boatto G (2013) Cross reactivities of 41 new amphetamine designer drugs to EMIT $^{\circledR}$ immunoassays. Forensic Toxicol 31:133-137

3. Uchiyama N, Shimokawa Y, Kawamura M, Kikura-Hanajiri R, Hakamatsuka T (2014) Chemical analysis of a benzofuran derivative, 2-(2-ethylaminopropyl)benzofuran (2-EAPB), eight synthetic cannabinoids, five cathinone derivatives, and five other designer drugs newly detected in illegal products. Forensic Toxicol 32:266-281

4. European Monitoring Centre for Drugs and Drug Addition (EMCDDA) (2012) Annual report on the state of the drugs problem in Europe. http://www.emcdda.europa.eu/publications/ annual-report/2012. Accessed 3 Nov 2014

5. Hill SL, Thomas SHL (2011) Clinical toxicology of newer recreational drugs. Clin Toxicol 49:705-719

6. European Monitoring Centre for Drugs and Drug Addition (EMCDDA) (2014) Risk assessment report of a new psychoactive substance: 3,4-dicholo- $N$-\{[1-(dimethylamino)cyclohexyl]methyl $\}$ benzamide (AH-7921) in the frame work of the Council Decision on new phychoactive substances. EMCDDA, Lisbon, pp 1-23 
7. Coppola M, Mondola R (2015) AH-7921: a new synthetic opioid of abuse. Drug Alcohol Rev 34:109-110

8. Hayes AG, Tyers MB (1983) Determination of receptors that mediate opiate side effects in the mouse. $\mathrm{Br} \mathrm{J}$ Pharmacol 79:731-736

9. Kronstrand R, Thelander G (2013) http://www.soft-tox.org/files/ Designer_Drugs/AH-7921_January_2013.pdf. Accessed 1 Nov 2014

10. Presidenza del Consiglio dei Ministri. Dipartimento Politiche Antidroga. Sistema Nazionale di Allerta Precoce. National early warning system (2013) 3,4-Dicloro- $N$-[(1-(dimetilammino) cicloesil) metil] benzamide (AH-7921). Italy, pp 1379-1385

11. Uchiyama N, Matsuda S, Kawamura M, Kikura-Hanajiri R, Goda Y (2013) Two new-type cannabimimetic quinolinyl carboxylates, QUPIC and QUCHIC, two new cannabimimetic carboxamide derivatives, ADB-FUBINACA and ADBICA, and five synthetic cannabinoids detected with a thiophene derivative $\alpha$-PVT and an opioid receptor agonist AH-7921 identified in illegal products. Forensic Toxicol 31:223-240

12. Cayman Chemical (2014) https://www.caymanchem.com/app/ template/Product.vm/catalog/12036/promo/emolecules. Accessed 5 Nov 2014

13. Cerilliant Corporation (2014) http://www.cerilliant.com/ShopOn line/Item_Details.aspx?itemno=98ce2a1d-a5dc-417b-b6147d4a2c8c747a. Accessed 5 Nov 2014

14. European Monitoring Centre for Drugs and Drug Addition (EMCDDA) (2014) AH-7921. EMCDDA-Europol joint report on a new psychoactive substance: AH-7921 3,4-dicholo- $N$-\{[1(dimethylamino)cyclohexyl]methyl\}benzamide. In accordance with Article 5 of council decision 2005/387/JHA on the information exchange, risk assessment and control of new psychoactive substances. EMCDDA, Lisbon

15. World Health Organization (2014) AH-7921. Critical review report. Agenda item 4.21. Expert committee on drug dependence. Thirty-sixth meeting. Geneva, 16-20 June 2014

16. Erowid (2013) AH-7921 (experience vaults). Extreme relaxation: an experience with AH-7921 (ID 93949) by Samuel. http://www. erowid.org/experiences/exp.php?ID=93949. Accessed 15 May 2014

17. Erowid (2014) AH-7921, alcohol \& cannabis by Selggurkim Citation: Selggurkim. "Ups downs AH town: an experience with AH-7921, alcohol \& cannabis (ID 102804)”. https://www.ero wid.org/experiences/exp.php?ID=102804. Accessed 14 May 2014

18. Bluelight (2014) http://www.bluelight.org/vb/threads/689803-TheAH-7921-Side-effect-and-withdrawal-WARNING. Accessed 15 May 2014

19. Tyers MB (1980) A classification of opiate receptors that mediate antinociceptive in animals. Br J Pharmacol 69:503-512

20. Brittain RT, Kellett DN, Neat ML, Stables R (1973) Anti-nociceptive effects in $N$-substituted cyclohexylmethylbenzamides. $\mathrm{Br}$ J Pharmacol 49:158-159
21. Sewell RDE, Spencer PSJ (1974) Biogenic amines and the antinociceptive activities of agents with a non-opiate structure. J Pharm Pharmacol 26(Supplement):92-93

22. Loew G, Lawson J, Toll L, Frenking G, Berzetei-Gurske I, Polgar W (1988) Structure activity studies of two classes of beta-aminoamides: the search for kappa-selective opioids. In: Harris LS (ed) Problems of drug dependence. Proceedings of the 50th annual scientific meeting. The committee on problems of drug dependence. NIDA Research Monograph 90, NIDA, Baltimore

23. Erowid (2013) For science AH-7921. http://erowid.org/experi ences/exp.php?ID=100939. Accessed 15 May 2014

24. Drugs-Forum (2014) http://www.drugs-forum.com/forum/showth read.php?t=234846\#ixzz31 gWqcyE7. Accessed 5 Nov 2014

25. Elliot S, Evans J (2014) A 3-year review of new psychoactive substances in casework. Forensic Sci Int 243:55-60

26. Vorce SP, Knittel JL, Holler JM, Magluilo J Jr, Levine B, Berran P, Bosy TZ (2014) A fatality involving AH-7921. J Anal Toxicol 38:226-230

27. Soh YN, Elliott S (2013) An investigation of the stability of emerging new psychoactive substances. Drug Test Anal 6:696-704

28. Uchiyama N, Matsuda S, Wakana D, Kikura-Hanakiri R, Goda Y (2013) New cannabimimetic indazole derivatives, $N$-(1-amino-3methyl-1-oxobutan-2-yl)-1-pentyl- $1 H$-indazole-3-carboxamide (AB-PINACA) and $N$-(1-amino-3-methyl-1-oxobutan-2-yl)-1-(4fluorobenzyl)- $1 H$-indazole-3-carboxamide (AB-FUBINACA) identified as designer drugs in illegal products. Forensic Toxicol 31:93-100

29. EMCDDA (2014) https://ednd.emcdda.europa.eu/html.cfm/ index7246EN.html?SUB_ID=258\&detail. Accessed 5 Nov 2014

30. Southern Association of Forensic Scientists (2014) http://fore ndex.southernforensic.org/index.php/detail/index/1249. Accessed 5 Nov 2014

31. Scientific Working Group for the Analysis of Seized Drugs (2014) http://www.swgdrug.org/Monographs/AH-7921.pdf. Accessed 5 Nov 2014

32. European Commission (2014) Proposal for a council decision on subjecting 4-iodo-2,5-dimethoxy- $N$-(2-methoxybenzyl)phenethylamine (25I-NBOMe), 3,4-dichloro- $N$ - $\{[1$-(dimethylamino)cyclohexyl]methyl $\} \quad$ benzamide (AH-7921), 3,4methylenedioxypyrovalerone (MDPV) and 2-(3-methoxyphenyl)2-(ethylamino)cyclohexanone (methoxetamine) to control measures. Brussels, 16.6.2014. COM(2014) 362 final. 2014/0183 (NLE)

33. Government of Israel (2014) http://www.health.gov.il/Legisla tionLibrary/Homarim-12092013.pdf. Accessed 5 Nov 2014

34. Anthony Gill, Delegate of the Secretary of the Department of Health, Australian Government (2014) Act 1989. Poisons standard amendment no. 2 of 2014. Australian Government, Camberra 